\section{RENAL HEMODYNAMIC CHANGES ASSESSED BY COLOR DOPPLER ULTRASOUND IN CHILDREN WITH LIVER CIRRHOSIS.}

\author{
N.H. Abufaddan ${ }^{1}$, N.N. Omar ${ }^{2}$, E. Mansour ${ }^{2}$ \\ ${ }^{1}$ Pediatrics, ${ }^{2}$ Diagnostic Radiology, Assiut \\ University, Assuit, Egypt
}

Background: Detection of early renal affection before proceeding to Hepatorenal failure as a complication of liver cirrhosis in children will be helpful in the prognosis of the disease.

The objective: Is to detect early hemodynamic changes in children with liver cirrhosis who had normal kidney function, to measure the pulsatility $(\mathrm{PI})$ and resistance $(\mathrm{RI})$ indices in children with different stages of liver cirrhosis, to compare them with apparently and their correlation to some risk factors (severity of cirrhosis, renal infection, blood hemoglobin level and the presence of portal hypertension).

Patients and methods: Thirty children with liver cirrhosis of different stages using Child- TurcottePaugh Score, all of them with normal kidney function tests as well as 10 apparently healthy children were included. PR and PI were measured by Doppler spectral wave analysis for patients and control.

Results: Both PI index and RI index are significantly higher in all children with liver cirrhosis compared with the control group. Both indices showed highest values with class $C$ compared with class $B$ and $A$. Each correlated significantly with anemia, renal infection and the presence of portal hypertension.

Conclusion: Monitoring of resistance indices (PIand RI) measured noninvasevely by Doppler ultrasound is an effective and easy method for detection of very early renal hemodynamic alternation in childhood cirrhosis and consequently early proper management. PI and RI were closely related to the severity of cirrhosis, renal infection, anemia and the presence of portal hypertension.

Keywords: Childhood cirrhosis. Renal hemodynamics. Duplex-Doppler ultrasonography

\section{HYPOURICEMIA IN THE COURSE OF NEOPLASTIC DISEASE OF CHILDREN}

\author{
A. Matsunaga, K. Toyota, M. Furuyama, \\ D. Sendo, T. Mitsui, K. Hayasaka
Pediatrics, Yamagata University School of Medicine, Yamagata, Japan

Urate can be an antioxidant and might provide immunological regulation. Hyperuricemia is regarded as the most important risk factor for the development of gout. Nevertheless, hypouricemia has rarely received attention from researchers. We previously studied 104 patients who were undergoing chemotherapy for neoplastic disease in our hospital during 2002-2006 and detected hypouricemia in 62 of them (59.6\%), particularly in patients with a solid tumor showing high mortality (42nd Japanese Pediatric Nephrology Congress Meeting 2007). No report describes effects of hypouricemia on the condition of the patients. Therefore, we studied the relation between clinical symptoms and hypouricemia during chemotherapy for neoplastic diseases in childhood.

We found 52 patients who had hypouricemia $(<2.0$ $\mathrm{mg} / \mathrm{dl}$ ) during hospitalization in our institute during February 2008 - October 2009. Of the 52 patients, 38 had neoplastic disease, 7 had neurologic diseases, 5 were newborns, and 2 were metabolic diseases. No patient had renal disease. Among the 38 patients with neoplastic disease were 21 reporting episodes of infectious diseases, and 17 patients who complained of digestive symptoms. They tended to have such episodes when the level of serum uric acid was low. It is noteworthy that eight patients died.

Patients with neoplastic diseases frequently have hypouricemia because of undernutrition and/or renal tubular damage attributable to treatment with antineoplastic agents. Hypouricemia might influence prognoses because of a lack of antioxidant or immunological regulation activities. 